\title{
Análisis de las técnicas documentales en las radios universitarias de España: Propuesta de Centro de Documentación
}

\author{
Agustín Vivas Moreno \\ Universidad de Extremadura, España \\ aguvivas@unex.es

\section{Daniel Martin-Pena} \\ Universidad de Extremadura, España \\ danielmartin@unex.es \\ Macarena Parejo Cuéllar \\ Universidad de Extremadura, España \\ macarenapc@unex.es
}

Cita sugerida: Vivas Moreno, A., Martin-Pena D. y Parejo Cuéllar, M. (2018). Análisis de las técnicas documentales en las radios universitarias de España: Propuesta de Centro de Documentación. Palabra Clave (La Plata), 7(2), e052.https://doi.org/10.24215/18539912e052 


\title{
Análisis de las técnicas documentales en las radios universitarias de España: Propuesta de Centro de Documentación
}

Analysis of the documentary techniques in the college radios of Spain: Proposed documentation Center

\author{
Agustin Vivas Moreno \\ Universidad de Extremadura, España \\ aguvivas@unex.es \\ Daniel Martin-Pena \\ Universidad de Extremadura, España \\ danielmartin@unex.es \\ Macarena Parejo Cuéllar \\ Universidad de Extremadura, España \\ macarenap@unex.es
}

\section{Resumen:}

La presente investigación tiene dos objetivos. Por un lado, analizar el empleo de las técnicas documentales utilizadas en las emisoras universitarias españolas pertenecientes a la Asociación de Radios Universitarias de España (ARU) y, por otro, confeccionar un modelo de Centro de documentación para dichas estaciones radiofónicas. Para el primer objetivo, la metodología utilizada ha sido de carácter cuantitativo, con un posterior análisis cualitativo de los datos obtenidos; para ello, se ha creado un instrumento validado y respondido por los responsables de las emisoras universitarias. Para el segundo, se ha seguido el método propiamente documental, con el fin de planificar un Centro de documentación específico para las radios universitarias, empleando, entre otros, el Sistema de Información promovido por la Secretaría de la UNESCO, que utilizamos fundamentalmente. Las principales conclusiones reflejan la casi nula importancia que prestan las emisoras a las labores de documentación, solventada en parte gracias a determinadas herramientas y repositorios localizados en la red en los cuales almacenar y catalogar de manera genérica el trabajo generado. Así mismo, la creación de un Centro de documentación de carácter colaborativo supondría la profesionalización de estas emisoras y la preservación de un patrimonio histórico de gran valor para la institución universitaria.

Palabras Clave: Centro de documentación, Radio, Universidad, Técnicas documentales.

\section{ABstRACT:}

The present research has two aims. On the one hand, to analyze the use of documentary techniques used in Spanish college radio stations belonging to the Association of University Radios of Spain (ARU), and on the other hand, to create a model of documentation center for these radio stations. The first objective is the methodology used has been quantitative, with a subsequent qualitative analysis of the data obtained; for it, an instrument validated and answered by the heads of university radio stations has been created. Secondly, the documentary method has been followed, in order to plan a specific documentation center for university radios, using for instance, the Information System promoted by the UNESCO Secretariat, that we use fundamentally. The main conclusions reflect the almost null importance of the stations to the documentation work, partially satisfied to certain tools and repositories located in the network in which to store and catalog generically the work generated. In the same way, the creation of a collaborative documentation center would mean the professionalization of these stations and the preservation of a valuable historical heritage for the university institution.

KEYWORDS: Documentation Center, Radio, University, Documentary techniques.

\section{INTRODUCCIÓN A LA IMPORTANCIA DE LA DOCUMENTACIÓN EN LA RADIO}

La radio, y nos referimos aquí a la generalista, es un medio de comunicación con un siglo de vida en España. A lo largo de su historia y también como consecuencia natural del desarrollo de las TIC la esfera radiofónica ha debido ir reinventándose y adaptándose a los nuevos tiempos.

En este sentido, si por algo se caracteriza el devenir de la radio ha sido por su capacidad para ir transmutando hacia un modelo más accesible, más autónomo (López Vidales, 2011) y con novedosos formatos para un 
consumo diferente (Ortiz Sobrino, 2011). Una situación favorecida, además, como es conocida, por el paso a la web semántica o web 3.0.

Al fin y al cabo, como subrayan Küster y Hernández, este entorno:

Tiene la capacidad de construir un cúmulo de conocimientos sobre las preferencias de los usuarios y que, a través de una combinación entre su capacidad de conocimiento y la información disponible en Internet, sea capaz de atender de forma exacta las demandas de información de los usuarios (Küster y Hernández, 2013, p. 106).

Sin embargo, y a pesar de que como sostienen Serra-Pallarés y Espinosa-Mirabet (2011) la documentación en el periodismo, en general, supone la marca que diferencia un producto profesional de otro amateur, los servicios de documentación sonoro constituyen una actividad muy joven. Algunos de los expertos que más han ahondado en la materia, como López-Yepes (1995), posicionan los inicios de la documentación en los medios de España, a finales de los años 80 y 90 del siglo XX.

En el ámbito radiofónico, sólo Radio Nacional de España (RNE), como emisora pública, ha tenido históricamente la obligación de conservar unas declaraciones que podían constituir, con el paso de los años, un patrimonio cultural, como luego se ha podido constatar. Sin embargo, el resto de radios comerciales han tardado muchos años en darse cuenta de la importancia que podía tener salvaguardar el material informativo que estaban produciendo.

De hecho, aunque en el año 1969 surge la primera asociación internacional para la conservación de archivos sonoros, IASA (International Asociation of Sound and Audiovisual Archive), con una finalidad sobre todo de índole protectora, lo cierto es que en España no será hasta la llegada de la democracia cuando se asienten y adquieran un papel destacado los llamados Departamentos de documentación en las empresas informativas, tal y como apuntan Nuño y Sánchez (1999).

$\mathrm{Y}$ es que no se puede negar que históricamente a los documentos sonoros no se les ha dado tanta importancia como a otro tipo de materiales como los impresos. Es una realidad que fueron los diarios impresos los primeros en contar con "documentalistas especializados en información que apoyaron y reforzaron con datos, documentos e investigaciones cualquier tipo de planteamiento informativo que preparaba un redactor" (Recio, 2015, p. 395).

En este orden de cosas, sí que es cierto que la marcada diferencia entre unos documentos y otros se ha debido principalmente a un problema de espacio. De ahí que en muchas empresas radiofónicas se haya optado por almacenar sólo aquello que se consideraba importante y descartar una serie de materiales que ya hoy sería difícil calcular su valor.

No obstante, tal y como venimos justificando, esta situación ha de ser entendida en el contexto radiofónico, donde la inmediatez por emitir la noticia y la necesaria rapidez por la búsqueda de información ha llevado irremediablemente a regrabar cintas como fórmula de reutilizar los recursos. Por todo ello, ha ocurrido que mientras la radio pública asumía el rol de ser la "memoria sonora histórica del país", las emisoras privadas guiaban su día a día sin ser plenamente conscientes de que en realidad estaban haciendo historia y, en consecuencia, no han mostrado una severa preocupación por preservar ese material.

De todo esto se deriva que, incluso en lo digital, el desarrollo de la gestión de la información en las entidades informativas públicas y privadas haya sido dispar. Así, Radio Nacional de España fue pionera en la digitalización de sus documentos sonoros, si bien no fue hasta el año 1998, para terminar cuatro años más tarde, cuando comenzó la digitalización de 1.400 .000 registros documentales y cerca de 190.000 horas de audio, 60.000 horas de música ligera, 52.000 de música clásica y tradicional, 60.000 horas de voces y programas informativos, 500 horas de efectos sonoros y 10.000 horas de obras de teatro y seriales radiofónicos. A partir de estos momentos, se digitalizarán entre 18.000 y 20.000 horas por año.

Frente al contexto radiodifusor público encontramos el espacio de índole comercial. En este sentido, otro estudio realizado en el año 2013 por Fernández-Sande, Rodríguez-Barba y Rodríguez-Pallares, donde se comparaban las principales cadenas de radiodifusión española (Cadena Ser, Onda Cero y Cope) con el 
objetivo de analizar cómo se estructuran en ellos los departamentos encargados de la gestión de contenidos, se pudo apreciar que los modelos de gestión son muy diferentes.

De los tres casos analizados Prisa Radio representa el modelo de gestión de contenidos más evolucionado. Dispone de un departamento de documentación consolidado en su estructura organizativa, capaz de desarrollar procesos de producción propia y difusión de información aportando de esa forma un valor añadido más visible a la oferta radiofónica. También se diferencia por haber desarrollado un CMS de creación propia adaptado a sus características y objetivos, Enciclomedia, que posibilita un modelo de trabajo en red Internacional (Fernández-Sande et al., 2013, p. 397).

El resto o bien han optado por contrataciones externas (COPE) o bien no parece que las empresas matrices decidan a corto plazo crear un departamento de gestión de contenidos propio (Onda Cero).

Realizada esta radiografía del panorama de la documentación informativa en España, y volviendo al caso de RNE, cuando sus archivos fueron presentados públicamente por el entonces director de RNE José Antonio Sentis, este dijo que la cadena pública "cuenta con uno de los archivos en español más importantes del mundo" y afirmó que con esta iniciativa, que consideró "pionera", se podría conservar "un patrimonio sonoro de gran valor" y testimonios "únicos".

Sin duda, y viendo la disparidad existente en cuanto a la gestión documental de la información, estas palabras nos sirven para explicar que el valor que adquiere la documentación en el medio radiofónico universitario es doble. Por un lado, supone un legado histórico, y por otro, resulta ser una apuesta por la creación de nuevos materiales informativos. Al final, documentar todo este material no es "simplemente seleccionar el fragmento de un discurso o una declaración aislada para incluirlo en un boletín o reportaje" (Nuño y Sánchez, 1999), sino que permite, además, crear un programa íntegramente con el material de archivo.

No debe perderse de vista que en las fonotecas de radio se suelen conservar diversos materiales. Es habitual hallar en ellos desde grabaciones originales (musicales o no musicales) a programas completos (elaborados por un presentador, productor, artista...), pasando por emisiones en directo, así como una amplísima colección de "cortes" o fragmentos editados con interés informativo, cultural, comercial y de todo tipo.

Estos suelen estar catalogados en función de diversas categorías (protagonista, tema...) y con ello se logra contar con un material ordenado en el que subyacen una serie de funciones que en el contexto de la radiodifusión vienen a cumplir una serie de funciones importantes (Galdós en Giménez, 2007):

- Función previsora: una parte de las informaciones que cada día recibimos es previsible. Se repite cíclicamente. Las alergias, la muerte o aniversario de ciertas figuras como Cervantes o conmemoraciones de días oscuros de nuestra historia (atentados, accidentes, etc.) son algunos de los hitos de los que es sencillo tener constancia. Sabemos que van a ocurrir. En este caso, la documentación permite a los periodistas anticiparse e ir trabajando en la elaboración de un material informativo que contribuye, sin duda, a la buena organización de su trabajo.

- Función preparatoria: hay géneros que precisan de cierta elaboración y documentación previa. Sobre todo la semblanza biográfica, el reportaje o la entrevista requieren de una búsqueda de información. Tener la documentación presente en estos casos marcará la diferencia entre lo superficial y lo profundo.

- Función crítico-verificadora: cualquier profesional de la comunicación está obligado a ofrecer informaciones rigurosas. Por eso, los profesionales de los medios de comunicación deberán dedicar una parte de su trabajo a la verificación de los datos que están ofreciendo.

- Función completiva: son muchos los datos que pueden convertir una simple información en una noticia completa. Acudir a los archivos puede servir para marcar la diferencia con respecto a la misma información emitida desde otros medios. 
- Función lingüístico-clarificadora: en este sentido, el Centro de documentación deberá proporcionar, además, toda la información necesaria sobre la correcta ortografía de una palabra, la expresión idónea para comunicar algo o la terminología adecuada.

- Función informativa: ha sido tradicional que los documentalistas colaborasen facilitando la información a los periodistas. Sin embargo, al día de hoy en muchos casos conocen tan bien los diferentes temas que, incluso, pueden ayudar a analizar ese contenido, lo que supone un valor añadido importante a su función.

En las radios, por tanto, entra en juego la figura de un nuevo profesional que debe ser aliado del periodista. El documentalista digital pasa a desarrollar nuevas destrezas encaminadas a la gestión de la documentación, la especialización en el manejo de herramientas informáticas, la elaboración de productos informativodocumentales, el empleo de los lenguajes documentales como fórmula para la búsqueda y recuperación de la información, la publicación de las ediciones digitales y la coordinación con el equipo de redacción (Rubio, 2005).

Ahora bien, ¿cuál es el contexto radiodifusor universitario en España? Sin extendernos en este punto, quizás convenga recordar aquí que el nacimiento de las radios universitarias en España se sitúa muy recientemente, en las últimas décadas del siglo XX. Nada que ver con la realidad en América, donde países como Argentina acogen las primeras emisiones en el año 1924 (Radio Universidad de La Plata). La primera emisora iniciadora de este movimiento es Radio San Fernando (actual Radio Campus) de la Universidad de La Laguna, que se pone en marcha en 1987. No obstante, lo cierto es que más de una década antes, concretamente en el año 1974, ya comienzan de manera regular las primeras grabaciones educativas procedentes de una institución de educación superior, la Universidad Nacional de Educación a Distancia, que utiliza las frecuencias de Radio Nacional de España en sus distintos canales para llegar a todo el país y, por ende, a todo su alumnado (Aguaded y Martín-Pena, 2013).

Sea como fuere, más allá de estos primeros inicios, lo cierto es que el apogeo de las radios universitarias, según sostienen Martín-Pena y Contreras (2014), se produce sobre todo a partir de los inicios del presente siglo. Esta proliferación tiene lugar como consecuencia natural del desarrollo de las TIC que permite a las instituciones establecer un diálogo más directo con la sociedad e, incluso, comenzar un trabajo colaborativo en red que aumenta las posibilidades comunicativas en materias como la divulgación científica o la formativa, entre otras.

Más allá del momento exacto de su surgimiento, un concepto que caracteriza a estos entes radiodifusores es la heterogeneidad. Esta particularidad marca una clara diferencia entre unas y otras. Sólo en el contexto radiodifusor universitario español es posible encontrar desde emisoras cuyo objetivo principal es ofrecer prácticas a los alumnos de titulaciones vinculadas con el mundo de la comunicación, a otras que resultan ser lugares de investigación y experimentación de nuevos formatos y altavoces con una clara vocación social hacia la ciudadanía. La radio universitaria, pues, como entidad que tiene la capacidad de congregar a la comunidad universitaria y no universitaria, no sólo como oyentes pasivos, sino como emisores activos y receptores participativos.

En la actualidad, en España existen 38 emisoras universitarias. Estas estaciones emiten tanto en FM, como en streaming o podcast. No obstante, los temas que cuentan con un mayor peso en las emisoras analizadas son los de música y entretenimiento, evidenciando que las radios universitarias seleccionadas no terminan de arriesgar en su oferta de programas diferenciadores. Como apunta Fidalgo (2009, p. 135), "las radios universitarias se encuentran en pleno proceso de búsqueda de identidad dentro del amplio espectro de los medios de comunicación".

Llegados a este punto, explicitemos las causas que obligan a la presencia de un Centro de documentación en el ámbito radiodifusor universitario. 


\section{UN CENTRO DE DOCUMENTACIÓN EN EL ÁMBITO RADIODIFUSOR UNIVERSITARIO}

Si la figura del documentalista es importante, naturalmente no lo es menos la del Centro de documentación. Autores como Recio (2015) afirman que este espacio tiene un valor sumamente importante para el periodista. En un estudio realizado por este autor se confirmaba que este centro es consultado de forma presencial por el $50 \%$ de los profesionales de la comunicación, y que hasta un $80 \%$ lo hace de forma online. Añade:

Además, la tasa de respuesta por parte de los documentalistas es muy rápida, ya que un $60 \%$ respondió que recibía la información en menos de una hora, lo que demuestra la eficacia de esos centros y la posibilidad que tiene el periodista de hacer una buena información al tener de manera rápida esos resultados. Por último, que los centros de documentación funcionan, lo deja claro esta cifra: el $40 \%$ señala que es bueno; aceptable y regular suman otro $47 \%$ y un $10 \%$ lo considera excepcional (Recio, 2015, p. 420).

Como puede comprobarse, en el marco de una emisora radiofónica esta herramienta tiene una trascendencia de primer nivel como suministrador de información. Por eso, es de deducir que si las funciones de este son relevantes en el contexto mediático no lo son menos en el institucional, donde cobran protagonismo departamentos de comunicación como son las radios universitarias.

Al fin y al cabo estas estaciones universitarias son, junto a los archivos, las bibliotecas, los centros de documentación universitarios, las revistas de divulgación científica, las redes sociales o la televisión universitaria, entre otras, instrumentos de información y comunicación al servicio de las instituciones de Educación Superior (Martín-Pena, Parejo y Vivas, 2016). Estas generan un volumen importante de información a través de entrevistas a investigadores sobre hallazgos científicos, cobertura de actos institucionales de relevancia, elaboración de programas por parte de científicos, estudiantes, etc., y, por ello, deben contar también con un sistema estratégico de recuperación, almacenamiento y gestión de la información que facilite una mayor difusión de la documentación sonora.

Cabe decir en este punto que aunque aquí utilicemos preferentemente el término Centro de documentación, podría ser en líneas generales sustituido por el de Servicio de documentación. Si bien existen diferencias conceptuales desde un punto de vista estricto, dado que comúnmente se entiende que el Centro debe estar integrado en un Servicio, como decimos aquí los utilizaremos indistintamente. Pero, ¿cuáles podrían ser las características del Centro de documentación referido?:

- En primer lugar, es el espacio donde se desarrollan tareas de documentación y de manejo y difusión de información en diversos grados (norma UNE 50/113 de conceptos fundamentales de documentación e información el término Centro de documentación);

- Así mismo, es el elemento intermedio necesario para reunir toda la información disponible en el tema de interés y, del mismo modo, organizarla, tratarla y suministrarla a sus usuarios en forma directamente utilizable.

- Del mismo modo, es el espacio en que para la gestión de la información de una radio universitaria debe hacer frente básicamente a diferentes tipos de tareas (Vivas y Martos, 2011):

a) funciones de entrada, acopio de los documentos que interesen a la estación universitaria. Las operaciones básicas son la selección, la adquisición y el registro de documentos;

b) funciones de tratamiento de la documentación, procedimientos que permiten representar el contenido de los documentos con el objeto de referenciar la información contenida y, en consecuencia, pueda ser recuperada en consulta y difundida posteriormente;

c) funciones de salida, esto es, elaboración de productos documentales adaptados a las demandas de los usuarios;

d) y por último, funciones de sustento con objeto de mantener y supervisar lo realizado. Incluye funciones administrativas, de gestión, de obtención de recursos, de evaluación, etc. 
Además, las alteraciones experimentadas a partir del desarrollo del periodismo digital permiten sobre todo la implementación de este tipo de estrategias en medios de comunicación institucionales como son las radios universitarias. En este sentido, el desarrollo de la red de redes representa una oportunidad única también para salvaguardar aquella información generada por un medio hecho sustancialmente por alumnos, profesores e investigadores. Sin embargo, opinamos como Rubio al decir que esto requiere de cierta planificación:

La evolución de los roles del servicio de documentación y de la redacción pretende forjar una nueva asociación informativa, en la que las habilidades de la documentación como evaluador, rastreador y entrenador de información cooperen con el papel de la redacción como intérprete, y escritor convincente (Rubio, 2005, pp. 153-168).

Precisamente, observando las posibilidades profesionales que la documentación ofrece al sector informativo, creemos necesario evaluar el peso que en la actualidad tiene en estas radios universitarias. La idea es, en este sentido, no sólo estudiar cómo se estructura en ellas esta disciplina sino, además, proponer un protocolo para la implementación de esta herramienta que se perfila, entre otros muchos aspectos, como un eficaz instrumento de patrimonio universitario.

\section{ObJetivos Y Metodología}

La investigación planteada tiene dos fases diferenciadas:

- La primera de ellas tiene por objeto analizar el empleo de las técnicas documentales utilizadas en las emisoras universitarias españolas que pertenecen a la Asociación de Radios Universitarias de España (ARU) (O1), asociación sin ánimo de lucro que integra a 29 estaciones radiofónicas universitarias que emiten desde universidades españolas, tanto públicas como privadas. Esta asociación se creó en el año 2011 con el propósito de compartir experiencias, formación o contenidos relacionados con la creación y la divulgación científica. A su vez, este objetivo se complementaría con otros cuatro de carácter más específico:

- examinar la presencia o no de documentalistas en las emisoras universitarias y determinar sus funciones; examinar la presencia o no de documentalistas en las emisoras universitarias y determinar sus funciones;

- estudiar los instrumentos de búsqueda y recuperación documental que utilizan las emisoras, y verificar si tienen instaurado un sistema automatizado de gestión documental;

- analizar el empleo de los lenguajes documentales en las emisoras universitarias, esto es, verificar cuál es el etiquetaje del material sonoro que hacen estas radios y cuál el modo de catalogar el patrimonio sonoro;

- por último, verificar cuál es el modo de archivar dicho patrimonio sonoro y la presencia o no de fonotecas especializadas.

Desde el punto de vista metodológico, para la consecución de estos objetivos, hemos planteado una investigación de carácter cuantitativo, con un posterior análisis cualitativo de los datos obtenidos. Para el acceso al campo de investigación hemos creado un instrumento concretado en un cuestionario estructurado en torno a seis preguntas, y que ha sido validado previamente por docentes del área de las Ciencias de la Información de distintas universidades españolas que poseen radio universitaria y conocedores de la realidad de las mismas. El cuestionario ha sido cumplimentado por los directores o coordinadores de las radios universitarias del estudio. Las preguntas que se plantearon en el cuestionario fueron las siguientes:

- ¿Existe la figura del documentalista en tu emisora universitaria? Si la respuesta es positiva, ¿quién realiza esa labor? y ¿qué tareas realiza? 
- ¿Existen instrumentos de búsqueda y recuperación de la información en la emisora? Si la respuesta es positiva, ¿cuáles?

- ¿Existe en la emisora un Sistema Automatizado de Gestión Documental?

- ¿Catalogáis los programas en tu radio?

- ¿Tenéis fonoteca en la emisora? Si la respuesta es positiva, ¿es una fonoteca tematizada o de programas?

- ¿Etiquetáis los programas en la emisora? Si la respuesta es positiva, ¿lo hacéis a través de palabras clave o descriptores normalizados?

Nuestra muestra está compuesta por un total de 24 radios universitarias, todas ellas pertenecientes a ARU. Fuera de la muestra quedan otras 5 universidades que tienen los proyectos radiofónicos en pausa o redefinición. Las emisoras que completan la muestra quedan recogidas en la Tabla 1, con la asignación de un código numérico que será utilizado en las tablas de resultados.

\begin{tabular}{|c|c|c|}
\hline $\begin{array}{c}\text { Emisora } \\
\text { Universitaria }\end{array}$ & Universidad & Código \\
\hline Radio UNED & UNED & 1 \\
\hline Radio Campus & Universidad de La Laguna & 2 \\
\hline InfoRadio UCM & Universidad Complutense & 3 \\
\hline Radio Salamanca & Universidad de Salamanca & 4 \\
\hline Radio Navarra & Universidad de Navarra & 5 \\
\hline Europea Radio & Universidad Europea Madrid & 6 \\
\hline UPV Ràdio & Universitat Politècnica València & 7 \\
\hline Vox UJI Ràdio & Universitat Jaume I & 8 \\
\hline OndaCampus Radio & Universidad de Extremadura & 9 \\
\hline Radio CEU Valencia & CEU Cardenal Herrera & 10 \\
\hline UniRadio Huelva & Universidad de Huelva & 11 \\
\hline UPF Ràdio & Universitat Pompeu Fabra & 12 \\
\hline Radio UMH & Universidad Miguel Hernández & 13 \\
\hline RUAH Alcalá & Universidad de Alcalá & 14 \\
\hline Radio URJC & Universidad Rey Juan Carlos & 15 \\
\hline iRadio UCAM & Universidad Católica de Murcia & 16 \\
\hline UAL Radio & Universidad de Almería & 17 \\
\hline UniRadio Jaén & Universidad de Jaén & 18 \\
\hline OnCEU Radio Lab & CEU San Pablo & 19 \\
\hline Radio.UniZar.es & Universidad de Zaragoza & 20 \\
\hline UNEAtlántico Radio & Universidad Europea Atlántico & 21 \\
\hline Radio Uva & Universidad de Valladolid & 22 \\
\hline Radio Politécnica & Universidad de Alicante & 23 \\
\hline UAB Ràdio & Universitat Autónoma Barcelona & 24 \\
\hline
\end{tabular}

TABLA 1

Muestra del estudio

Elaboración Propia

- En la segunda fase de nuestro estudio, y tras el análisis pertinente de los resultados, nuestra investigación tendrá por objeto confeccionar un modelo de Centro de documentación para las radios universitarias (O2). Dicho objetivo se verá especificado mediante dos fines específicos:

- Por un lado, establecer las diferentes fases de implementación de un Centro de documentación para las radios universitarias;

- Por otro, proyectar un Centro de documentación colaborativo que aglutine a las diferentes emisoras radiofónicas universitarias en el marco de la Asociación de Radios Universitarias de España (ARU).

Para todo ello hemos utilizado diferentes criterios: 
- Por un lado, el método descriptivo, utilizando la encuesta consensuada, y que persigue el análisis de los datos obtenidos y la extracción del panorama documental de las radios universitarias;

- Por otro, el método propiamente documental, con el objeto de planificar un Centro de documentación específico para las radios universitarias. Para ello, el modelo realizado es propio, si bien nos han resultado provechosos el Sistema METRICA, que es el Método para el Desarrollo de Sistemas de Información promovido por el Consejo Superior de Informática, el Manual para el Establecimiento de Unidades Documentales y Bases de Datos Bibliográficos Nacionales para la Política Científica y Tecnológica confeccionado por consultores y la Secretaría de la UNESCO, y algunos otros modelos no pensados para Centros de Documentación sino para la planificación de otras unidades de información. De todos ellos, posiblemente sea el modelo de la UNESCO el que fundamentalmente hemos utilizado de referencia, si bien ha sido debidamente actualizado.

\section{ANálisis de Resultados y discusión: El EMPleo de las tÉcnicas documentales UTILIZADAS EN LAS EMISORAS UNIVERSITARIAS ESPAÑOLAS}

En lo correspondiente a O1, mediante el proceso metodológico recogido en el apartado anterior, hemos obtenido una serie de resultados que nos permiten alcanzar los fines propuestos en nuestra primera fase de la investigación, esto es, la situación actual en el empleo de las técnicas documentales utilizadas en las emisoras universitarias españolas.

Los resultados quedan agrupados en dos tablas. Primeramente, en la Tabla 2 podemos observar los parámetros de análisis: presencia de documentalistas en las emisoras universitarias, uso de instrumentos de búsqueda y recuperación de la información y creación de sistemas de gestión documental automatizada, propios de las estaciones radiofónicas.

\begin{tabular}{|c|c|c|c|c|c|c|}
\hline \multirow{2}{*}{$\begin{array}{l}\text { Código } \\
\text { Radio } \\
\end{array}$} & \multicolumn{2}{|c|}{ Documentalista } & \multicolumn{2}{|c|}{ Instr. Búsqueda } & \multicolumn{2}{|c|}{ Sist. Ges. Doc. Automatizada } \\
\hline & SI & NO & SI & NO & SI & NO \\
\hline 1 & $\mathrm{X}$ & & $\mathrm{X}$ & & $\mathrm{X}$ & \\
\hline 2 & & $\mathrm{x}$ & $\mathrm{x}$ & & & $\mathrm{x}$ \\
\hline 3 & & $\mathrm{X}$ & $\mathrm{X}$ & & & $\mathrm{X}$ \\
\hline 4 & & $\mathrm{x}$ & $\mathrm{x}$ & & $\mathrm{x}$ & \\
\hline 5 & & $\mathrm{X}$ & $\mathrm{X}$ & & $\mathrm{X}$ & \\
\hline 6 & & $\mathrm{x}$ & & $x$ & & $x$ \\
\hline 7 & & $\mathrm{X}$ & $\mathrm{X}$ & & & $\mathrm{X}$ \\
\hline 8 & & $\mathrm{X}$ & $\mathrm{x}$ & & & $\mathrm{x}$ \\
\hline 9 & $\mathrm{x}$ & & & $\mathrm{X}$ & & $\mathrm{X}$ \\
\hline 10 & & $\mathrm{x}$ & $\mathrm{x}$ & & & $\mathrm{x}$ \\
\hline 11 & $\mathrm{X}$ & & $\mathrm{X}$ & & $\mathrm{X}$ & \\
\hline 12 & & $\mathrm{x}$ & $\mathrm{x}$ & & $\mathrm{x}$ & \\
\hline 13 & & $\mathrm{X}$ & $\mathrm{X}$ & & $\mathrm{X}$ & \\
\hline 14 & & $\mathrm{X}$ & & $\mathrm{X}$ & & $\mathrm{X}$ \\
\hline 15 & & $\mathrm{X}$ & $\mathrm{X}$ & & & $x$ \\
\hline 16 & & $\mathrm{x}$ & $\mathrm{x}$ & & & $x$ \\
\hline 17 & & $\mathrm{X}$ & $\mathrm{X}$ & & & $\mathrm{X}$ \\
\hline 18 & $\mathrm{X}$ & & & $\mathrm{x}$ & & $x$ \\
\hline 19 & & $\mathrm{X}$ & $\mathrm{X}$ & & & $\mathrm{X}$ \\
\hline 20 & & $x$ & & $\mathrm{X}$ & & $x$ \\
\hline 21 & & $x$ & & $\mathrm{X}$ & & $x$ \\
\hline 22 & & $\mathrm{X}$ & & $\mathrm{X}$ & & $\mathrm{X}$ \\
\hline 23 & $\mathrm{X}$ & & $\mathrm{X}$ & & & $x$ \\
\hline 24 & $x$ & & $\mathrm{x}$ & & & $x$ \\
\hline TOTAL & $25 \%$ & $75 \%$ & $71 \%$ & $29 \%$ & $25 \%$ & $75 \%$ \\
\hline
\end{tabular}

TABLA 2

Documentalista, Instrumentos búsqueda y Sist. Ges. Doc. Automatizada elaboración propia 
Sistematicemos los resultados obtenidos:

a) En las radios universitarias españolas la presencia de personal destinado a la documentación es prácticamente nula. Tan sólo seis emisoras (Radio UNED, UniRadio Huelva, UniRadio Jaén, Radio Politécnica, OndaCampus, UAB Ràdio) tienen alguna persona dedicada a estas labores. Y sólo la UNED tiene en plantilla un documentalista contratado. En las otras cinco estaciones son los alumnos en prácticas, o el propio personal de la emisora, los que tienen asignadas estas funciones. En este sentido, conviene reseñar un estudio reciente de Pinto, Martín-Pena y Vivas (2016, p. 226) que sostiene que los alumnos que cursan los estudios de Información y Documentación pueden "encontrar en la radio universitaria un lugar en el que desarrollar los conocimientos teóricos alcanzados en las aulas y poder adquirir competencias transversales útiles para su desarrollo profesional". En la muestra de nuestro estudio, hay un total de cinco universidades que poseen estudios relacionados directamente con las ciencias de la documentación (Grado de Información y Documentación o estudios de doctorados); estas instituciones son: Universidad Complutense, Universidad de Extremadura, Universidad de Zaragoza, Universidad de Salamanca y Universidad Autónoma de Barcelona. De estas cinco, sólo Extremadura y la Autónoma de Barcelona tienen a alumnos de esta área realizando tareas documentales en el seno de la emisora. En cuanto a las labores que realizan, los directores resaltan: la búsqueda de información y la gestión de archivo, fonoteca y documentación. Otros apuntan que es una labor multitarea, y coordinadores de emisoras que no tienen en su plantilla la figura del documentalista apuntan que, en realidad, son los propios redactores los que actúan como documentalistas en la fase de preparación de sus programas radiofónicos.

b) En cuanto a los instrumentos de búsqueda y recuperación de la información, 17 directores declaran que su emisora hace uso de herramientas de este tipo. A través de las respuestas, hemos detectado que en la mayoría de los casos no son sistemas propios sino que las emisoras, en el contexto de apogeo y desarrollo constante de las TIC, hacen uso habitual de las redes sociales de audio (iVoox o SoundCloud) para archivar sus programas y recuperarlos mediante búsquedas sencillas, además de conservar un sistema digital propio. En este sentido, tan sólo seis emisoras han desarrollado un sistema de gestión documental automatizada: Radio UNED, Radio Salamanca, Radio Navarra, UniRadio Huelva, UPF Ràdio y Radio UMH. Destaca Radio Navarra, que ha implementado una base de datos para la gestión de la programación que permite describir los archivos tomando directamente datos como la fecha y el nombre de programa, a los que se puede añadir una descripción temática, de autores e incluso una fotografía. El éxito del mismo depende, en buena medida, de quién gestiona el contenido sonoro y también de la información que los propios alumnos aporten. Por su parte, Radio UNED tiene desarrollado un sistema integrado en su Intranet, y UPF Ràdio se nutre de otro sistema desarrollado por la Unidad de Informática de su universidad que permite de manera automática la búsqueda de la información, tanto en los soportes físicos de la mediateca como en los recursos virtuales que tiene adscritos la universidad.

Finalmente, la Tabla 3 recoge otros parámetros analizados: la catalogación de los programas; el uso de tags o etiquetas en la descripción de los mismos y la creación de fonotecas accesibles para conservar el patrimonio sonoro. 


\begin{tabular}{|c|c|c|c|c|c|c|c|c|}
\hline \multirow{2}{*}{$\begin{array}{l}\text { Código } \\
\text { Radio }\end{array}$} & \multicolumn{3}{|c|}{ Fonoteca } & \multicolumn{2}{|c|}{ Catalogación Prog. } & \multicolumn{3}{|c|}{ Etiqueta programas } \\
\hline & NO & PRG. & TEM. & SI & NO & NO & PAL. & DESCRIP \\
\hline $\mathbf{1}$ & & $\mathrm{x}$ & & $\mathrm{x}$ & & & $\mathrm{x}$ & \\
\hline 2 & & & $\mathrm{x}$ & $\mathrm{x}$ & & & $\mathrm{x}$ & \\
\hline 3 & & $\mathrm{x}$ & & $\mathrm{x}$ & & & $\mathrm{x}$ & \\
\hline 4 & & $\mathrm{x}$ & & $\mathrm{x}$ & & & $\mathrm{x}$ & \\
\hline 5 & & $\mathrm{x}$ & & $\mathrm{x}$ & & & & $\mathrm{x}$ \\
\hline 6 & $x$ & & & $x$ & & & $x$ & \\
\hline 7 & & $\mathrm{x}$ & & $\mathrm{x}$ & & & $\mathrm{x}$ & \\
\hline 8 & & $\mathrm{x}$ & & & $\mathrm{x}$ & & $\mathrm{x}$ & \\
\hline 9 & & $\mathrm{x}$ & & $\mathrm{x}$ & & & $\mathrm{x}$ & \\
\hline 10 & & $\mathrm{x}$ & & & $\mathrm{x}$ & $\mathrm{x}$ & & \\
\hline 11 & & & $\mathrm{x}$ & $\mathrm{x}$ & & & $\mathrm{x}$ & \\
\hline 12 & & & $\mathrm{x}$ & $\mathrm{x}$ & & & $\mathrm{x}$ & \\
\hline 13 & & $\mathrm{x}$ & & $\mathrm{x}$ & & & $\mathrm{x}$ & \\
\hline 14 & $\mathrm{x}$ & & & & $x$ & $x$ & & \\
\hline 15 & & $\mathrm{x}$ & & $\mathrm{X}$ & & & $\mathrm{X}$ & \\
\hline 16 & & $x$ & & $x$ & & & $x$ & \\
\hline 17 & & $\mathrm{x}$ & & $\mathrm{x}$ & & & $\mathrm{x}$ & \\
\hline 18 & & $\mathrm{x}$ & & $x$ & & & & $\mathrm{x}$ \\
\hline 19 & & $x$ & & $x$ & & & $\mathrm{x}$ & \\
\hline 20 & & $x$ & & $x$ & & & $x$ & \\
\hline 21 & $x$ & & & & $x$ & & $x$ & \\
\hline 22 & $x$ & & & $x$ & & & $x$ & \\
\hline 23 & & $\mathrm{x}$ & & $\mathrm{x}$ & & & $\mathrm{X}$ & \\
\hline 24 & & $x$ & & $x$ & & & & $x$ \\
\hline$\%$ & $17 \%$ & $71 \%$ & $12 \%$ & $\mathbf{8 3} \%$ & $17 \%$ & $8 \%$ & $79 \%$ & $13 \%$ \\
\hline
\end{tabular}

TABLA 3

Fonoteca, Catalogación y Etiquetas Programas

elaboración propia

a) Si observamos la tabla podemos detectar que la catalogación de los programas es una tarea habitual en las estaciones universitarias, aunque la mayoría de directores declara que es una función realizada de manera muy intuitiva, al no disponer de especialistas en esta área en las plantillas de las estaciones.

b) En cuanto al desarrollo de fonotecas o audiotecas los directores consideran que es de suma importancia conservar el patrimonio sonoro. Incluso algunos declaran que tienen un archivo digital con toda la programación registrada desde la fundación de la emisora. Manifiestan que las TIC han sido unas grandes aliadas. Tan sólo cuatro emisoras no tienen desarrollados este tipo de recursos. En cuanto al tipo de fonoteca hemos detectado dos formatos. Por un lado, "la audioteca de programas", que representa el formato más sencillo de desarrollar por su operatividad (se acaba un programa, se registra y se archiva), esta opción es utilizada por la mayoría de emisoras (17 en total). Por otro lado, existe en tan sólo tres emisoras (UniRadio Huelva, UPF Ràdio y Radio Campus) "la fonoteca tematizada", la cual requiere una tarea más laboriosa, ya que precisa la escucha detenida de programas y su clasificación por temática. En este sentido, destaca la fonoteca de UniRadio Huelva, desarrollada a través de un programa de Innovación Docente y que ha sido reconocida en el año 2010 con Premio a la Innovación Docente de Excelencia de la Universidad de Huelva por el proyecto "Radioteca: recursos sonoros libres" (www.uhu.es/uniradio).

c) Un último parámetro de análisis es el etiquetaje de los programas, fundamental para su búsqueda y recuperación, así como para su posicionamiento en la web. Sólo Radio CEU y RUAH Alcalá no realizan esta función. El resto de las emisoras sí etiquetan sus audios. Existen dos formas diferenciadas. La intuitiva a través de palabras clave es la opción mayoritaria: la realizan 19 emisoras. Y la opción más correcta que sería a través de descriptores normalizados, que sólo realizan tres estaciones. 


\section{Implantación de UN MOdelo de CENTRo de documentaCión}

A la luz de los resultados se aprecia que, aunque existe cierta conciencia en cuanto a la gestión de la información -sobre todo a través de tags, etiquetas, repositorios-, las emisoras carecen en su totalidad de una estrategia de documentación. En este sentido, no sólo no es, hasta el momento, seña de identidad de las radios, sino que además ni siquiera es habitual encontrar al profesional de la documentación formando parte del equipo en plantilla o como colaborador de estos medios de comunicación universitarios. La gran variedad de públicos que la hacen posible, unido en muchos casos a la falta de recursos o de cimientos sólidos de supervivencia, han hecho que desde su surgimiento estas labores hayan estado supeditadas a un segundo plano. De hecho, según los datos expuestos, en el mejor de los casos, cuando se contemplan estas labores, suelen ser asignadas a alumnos en prácticas, no siempre del ámbito de la documentación, que además desarrollan su actividad bajo una filosofía multitarea.

Ante un panorama como el que hemos podido apreciar por los resultados recabados, y que describíamos en el párrafo anterior, se torna preciso abordar una segunda fase de estudio $(\mathrm{O} 2)$ consistente en la planificación, visto los datos obtenidos, de Centros de documentación adaptando, para ello, el modelo establecido por Vivas y Martos (2011, pp. 65-81). Además, dados los buenos resultados obtenidos hasta ahora en la colaboración en red establecida por estas emisoras, parece lógico pensar que este primer establecimiento podría contribuir a la creación de plataformas de gestión informativa conjuntas en el marco de la Asociación de Radios Universitarias.

La Tabla 4 muestra de forma sintética el proceso que sostenemos debe seguirse para alcanzar la implementación de un Centro de documentación en el seno de una radio universitaria, y que describiremos más adelante:

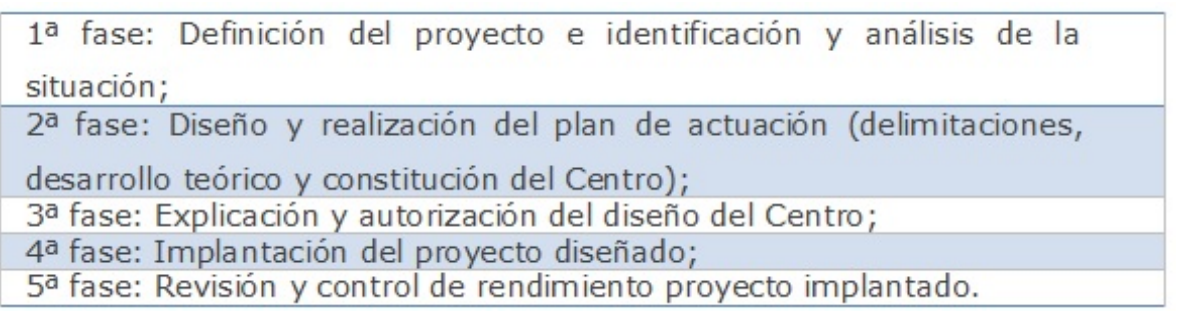

TABLA 4

Fase de implementación de un Centro de documentación Vivas y Martos (2011, pp. 65-81)

- Primera etapa: Definición del proyecto e identificación y análisis de la situación

En esta primera etapa sería preciso realizar un análisis de la situación de la radio universitaria en cuestión. Esta parte permitirá conocer las necesidades y potencialidades de cada emisora, esto es, el número de programas, las temáticas, el número de colaboradores, la estructura jerárquica de la emisora, etc. Así, y en base a ello, sería viable definir un proyecto más personalizado de Centro de documentación universitaria.

Como subraya Vivas y Martos (2011, p. 65) "el objetivo de este punto es asimilar un conocimiento del marco legal y jurídico y cualquier contorno normativo interno que pudiera resultarnos útil, como directrices generales, manuales de normas de organización, sistemas de calidad, catálogo de puestos y funciones, etc.”.

Además, este Centro debe proponerse no sólo como una herramienta para la radio universitaria sino más bien como un instrumento de índole institucional que potenciará la imagen de marca y a la identidad universitaria.

- Segunda etapa: Diseño y realización del Plan de actuación 
En esta segunda fase comenzará el diseño propiamente del proyecto. Por un lado, se tratará de delimitar su desarrollo y, por otro, se intentará determinar tanto la organización como la estructura que caracterizará a este Centro de documentación radiofónica universitaria. En este sentido, a la hora de delimitar el diseño del Centro de documentación deberán tenerse en cuenta los siguientes parámetros:

a) En primer lugar habrá que definir los objetivos, que no son otros que los que se enumeran a continuación:

- Constituir un sistema de información en el marco de la realidad radiofónica de la institución universitaria, con el propósito de satisfacer las necesidades informativas de las radios universitarias.

- Disponer de un servicio de referencia y orientación especializado en contenidos radiofónicos de índole universitaria.

- Establecer una gestión informativa en red entre las emisoras y el resto de los servicios y departamentos tanto externos como internos que integran la realidad universitaria. Este último propósito deberá ser acometido en una segunda fase, una vez existan proyectos en todo el tejido radiodifusor universitario.

b) Del mismo modo será preciso delimitar las temáticas: contenidos históricos, vertientes educativas y didácticas, culturales, biblioteconómicas, etc., esto es, la radio como fenómeno transversal al conjunto de las diversas disciplinas.

c) Asimismo, en esta primera toma de contacto con el proyecto de gestión documental será fundamental definir qué recursos son imprescindibles, tanto personales, materiales, tecnológicos y metodológicos para su puesta en marcha.

d) Igualmente, habrá que delimitar los servicios a prestar, que consistirían en:

- Difusión de documentos primarios, los documentos originales y completos;

- Publicación de documentos secundarios, es decir, abstracción de documentos primarios;

- Difusión Selectiva de la Información (DSI), esto es, el envío automatizado periódico de informaciones bibliográficas a usuarios abonados y caracterizados por sus perfiles de interés;

Una vez delimitado el Centro, deberán definirse algunos dispositivos organizativos como los que se destacan a continuación:

a) El establecimiento de un organigrama, donde se delimiten claramente cuáles serán las funciones del Centro de documentación dentro de la radio universitaria.

b) Redacción de una normativa interna o reglamento que sea presentado a la dirección para su aprobación, donde se especifican objetivos, servicios y recursos. Este debe recoger, entre otros aspectos, la dinámica de trabajo que deben seguir tanto el personal en plantilla como los colaboradores de la radio para salvaguardar el material que se vaya generando.

c) Del mismo modo será preciso cuantificar y presupuestar el proyecto. Se deberá lograr establecer un presupuesto y un estudio de viabilidad, teniendo en cuenta los recursos existentes en las radios universitarias y los necesarios para la implantación.

d) Generación de un cronograma del proceso de implantación que se ha llevado a cabo, especificando las fases de implantación en calendario.

Es recomendable que una vez realizada toda la parte de modelaje teórico trazado, todo el proyecto y los planteamientos de este se plasmen en un documento para su aprobación por las autoridades competentes. 
- Tercera etapa: Explicación y autorización del diseño

Será fundamental que este diseño de Centro de documentación universitario sea consensuado tanto dentro de la emisora (en un primer momento) como entre quienes constituyen la comunidad universitaria. Además, sería ideal que el proyecto antes de su aprobación fuera debatido en Consejo de Gobierno, de forma que fuese concebido como una herramienta necesaria y de utilidad por la comunidad en su conjunto. La idea es que se aprecie y valore su rentabilidad, su capacidad para apoyar tanto la docencia como la investigación, y sea visto a la vez como un recurso útil y de ahorro de tiempo para el propio medio universitario. Además, deberá ser percibido como una herramienta de patrimonio histórico de la institución así como un instrumento de marca.

- Cuarta etapa: Implantación del proyecto

En este periodo tendrá que desarrollarse la difusión del proyecto, pero acompañado de una serie de tareas:

1. Es importante que aquellas personas que vayan a gestionar este centro realicen algunos cursos específicos. En este sentido, opinamos que las personas que deben implementar el Centro de documentación en una radio universitaria deben reunir dos condiciones: por un lado, deben tener estudios esencialmente de Documentación, dado que deberán utilizar las técnicas y procedimientos documentales de forma eficiente; por otro lado, también deben conocer las funciones y objetivos que toda radio universitaria persigue, conociendo sus tareas y modelos de funcionamiento.Es importante que aquellas personas que vayan a gestionar este centro realicen algunos cursos específicos. En este sentido, opinamos que las personas que deben implementar el Centro de documentación en una radio universitaria deben reunir dos condiciones: por un lado, deben tener estudios esencialmente de Documentación, dado que deberán utilizar las técnicas y procedimientos documentales de forma eficiente; por otro lado, también deben conocer las funciones y objetivos que toda radio universitaria persigue, conociendo sus tareas y modelos de funcionamiento.

2. En este periodo deberán asignarse funciones y establecerse nuevas dinámicas de trabajo con el personal de la emisora. Hemos de tener en cuenta que en la mayoría de los casos estas emisoras han estado operando sin esta infraestructura, categorizando en función de los conocimientos de quienes en ella trabajan (con escasa presencia de documentalistas).

3. Aquí ya sí comenzará el tratamiento informativo de aquellos nuevos materiales y recursos que se vayan generando. Veamos cuáles son, siguiendo para ello las aportaciones de López Yepes y de Valle Gastaminza en Vivas y Martos (2011):

a) En primer lugar contamos con las operaciones de entrada, que constituirán los depósitos bibliográficos y documentales vinculados a la temática. Para ello, hay que seleccionar y adquirir los fondos y almacenarlos de forma registrada, clasificada y ordenada.

b) En segundo orden se encuentran las operaciones de tratamiento de la documentación. Se trata de aquellas tareas que están encaminadas a referenciar el contenido de los documentos: por un lado, descripción física del documento, es decir, catalogación; y, por otro, la descripción de su contenido para su ordenación y localización, y para su conocimiento e información.

c) En tercera instancia será fundamental diseñar operaciones de salida. Naturalmente su misión es esencial, pues tiene por objeto comunicar al potencial usuario la información pertinente que necesita. 
- Quinta fase: Revisión y control del rendimiento

Cuando esté ya desarrollado el proyecto de Centro de documentación para la emisora universitaria habrá llegado el momento, como es lógico, de revisar constantemente el correcto funcionamiento. Cada cierto tiempo será recomendable ejecutar un análisis de los datos recogidos, especificándose las debilidades o elementos a intensificar, las amenazas o elementos problemáticos, las fortalezas o elementos seguros y las oportunidades o elementos potenciales (matriz DAFO).

Además, una vez que cada emisora tenga constituida su propia herramienta de gestión documental, se podrá aspirar a un proyecto más amplio de tipo colaborativo que aglutinará a las diferentes emisoras universitarias que forman la ARU. Al contar con protocolos similares a través de herramientas tecnológicas será más fácil trabajar con un centro en red de tipo colaborativo.

\section{Conclusiones}

a) Tras la radiografía realizada en el seno de las radios universitarias, se pone de manifiesto la escasa o casi nula importancia que prestan estas a las labores de documentación. A pesar de las ventajas ya citadas acerca de la implementación de las labores de recuperación de la información en el contexto radiofónico, lo cierto es que la existencia de gestores documentales propios no es en estos momentos, salvo algunas excepciones, una preocupación para estos medios de comunicación. Estas labores recaen en su mayor parte en personal propio de la emisora (técnicos audiovisuales, redactores), en becarios e inclusive en estudiantes en prácticas que no pueden dedicarle el tiempo necesario ni la habilidad precisa para poner en valor la importancia del trabajo de documentación en el seno de una emisora.

b) Para estas estaciones se concluye que las necesidades de índole documental se solventan en la red, donde encuentran algunas herramientas y repositorios en los cuales almacenar y catalogar de manera genérica el trabajo generado. Lógicamente, estar supeditados a estas unidades de gestión externas disminuye posibilidades al proceso de selección, recogida, análisis, almacenamiento, recuperación y difusión la información. Resta autonomía a un medio relativamente joven y que, sin embargo, podría servirse de su condición de ente de conocimiento para propiciar la puesta en marcha de un Centro de documentación propio como el que aquí se plantea.

c) Finalmente, se propone la creación de un centro de documentación de carácter colaborativo. Tras la planificación expuesta, este centro supondría la profesionalización de una herramienta comunicativa universitaria como es la radio y la preservación de un patrimonio histórico de gran valor para la institución universitaria. Además, y dado el panorama que ha quedado reflejado donde muchas de ellas trabajan en colaboración (ARU), esta iniciativa permitiría extrapolar los resultados a un proyecto más ambicioso de carácter interuniversitario de documentación en red.

\section{ReFERENCIAS}

Aguaded, I., y Martín-Pena, D. (2014). Educomunicación y radios universitarias: panorama internacional y perspectivas futuras. Chasqui. Revista Latinoamericana de Comunicación, 124, 66-72.

Fernández-Sande, M., Rodríguez-Barba, D., y Rodríguez-Pallares, M. (2013). La gestión de contenidos como actividad estratégica en empresas de radiodifusión. Estudio de casos en la radio comercial española. El profesional de la información, 22(5), 392-398.

Fidalgo, D. (2009). Las radios universitarias en España. Transformación al mundo digital. Telos, 80.

Giménez, E. (2007). Manual de documentación para comunicadores (2a. ed.). Navarra: Eunsa.

Küster, I., y Hernández, A. (2013). De la Web 2.0 a la Web 3.0: antecedentes y consecuencias de la actitud e intención de uso de las redes sociales en la web semántica. Universia Business Review, 37, 104-119. 
López Vidales, N. (2011). La radio se transforma: nuevas tecnologías, nuevos hábitos y nuevos perfiles para el medio más cercano. En M. A. Ortiz Sobrino y N. López Vidales (eds.), Radio 3.0. Una nueva radio para una nueva era. La democratización de los contenidos (pp. 15-40). Madrid: Fragua.

López-Yepes, J. (1995). La documentación como disciplina. Teoría e historia. Pamplona: Eunsa.

Martín-Pena, D., Parejo, M., y Vivas, A. (2016). La radio universitaria: Gestión de la información, análisis y modelos de organización. Barcelona: Gedisa.

Martín-Pena, D., y Contreras Pulido, P. (2014). Las radios universitarias en España: inicios, evolución y panorama actual. En D. Martín-Pena, y M.A. Ortiz Sobrino (Coords.), Radios Universitarias en América y Europa (pp. 88-100). Madrid: Fragua.

Nuño, M.V., y Sánchez, M. (1999). Los servicios de documentación sonora ante el reto digital. Cuadernos de documentación multimedia, 8.

Ortiz-Sobrino, M. A. (2011). La radio española en el umbral de la web 3.0. En M.A. Ortiz Sobrino, y N. López Vidales (Eds.), Radio 3.0. Una nueva radio para una nueva era. La democratización de los contenidos (pp. 41-66). Madrid: Fragua.

Pinto, R., Martín-Pena, D., y Vivas, A. (2016). La radio universitaria como formadora de profesionales. Análisis de la relación entre los títulos de grado y la radio universitaria española. Revista General de Información y Documentación, 26(1), 221.

Recio, J. C. (2015). Análisis de la nueva perspectiva de la documentación periodística en los medios de comunicación españoles. Revista General de Información y Documentación, 25(2), 389-423.

Rubio, M. (2005a). A new age of documentation in online journalism: old and new functions of digital media library. Communication \& Society, 18(1), 153-168.

Rubio, M. (2005b). El perfil triangular del documentalista digital: novedades y constantes del emisor de documentación en el periodismo digital. Scire, 11(2), 17-32.

Serra-Pallaré, M., y Espinosa-Mirabet, S. (2011). La documentación en los estudios de comunicación: aplicación de herramientas 2.0. El profesional de la información, 20(2).

Vivas, A., y Martos, A. (2011). Modelo teórico de centros de documentación: el ejemplo del Centro de Documentación de estudios de Lectura y Escritura como modelo de gestión del saber interuniversitario. Revista Mexicana de Ciencias de la Información, 2(1), 65-81. 